\title{
Rare peritoneal tumour presenting as uterine fibroid
}

\author{
Janu Mangala Kanthi*, Sarala Sreedhar, Indu R. Nair
}

Department of Obstetrics \& Gynaecology, Amrita Institute of Medical Sciences, Ponekkara, Kochi-41, Kerala, India

Received: 21 February 2014

Accepted: 23 March 2014

\section{*Correspondence:}

Dr. Janu Mangala Kanthi,

E-mail: janu_durai@yahoo.com

(c) 2014 Kanthi JM et al. This is an open-access article distributed under the terms of the Creative Commons Attribution Non-Commercial License, which permits unrestricted non-commercial use, distribution, and reproduction in any medium, provided the original work is properly cited.

\begin{abstract}
Tumour arising from the uterus in the reproductive age group commonly belongs to benign category, the myoma uterus. Here we present a case report of a $22 \mathrm{yr}$ old primiparous mother with uterine metastasis from a rare peritoneal malignancy desmoplastic small round cell tumour who presented as uterine myoma. Desmoplastic small round cell tumour is a rare aggressive neoplasm arising from mesothelial cell, with poor prognosis. This woman presented with excessive bleeding per vaginum in the postpartum period,3months after caesarean section.24weeks enlarged uterine mass found for which uterine artery embolisation was done in vain, later she was planned to undergo myomectomy. But due to widespread uterine mass, hysterectomy was done which led to the diagnosis of this rare neoplasm.
\end{abstract}

Keywords: Desmoplatic small round cell tumour, Myoma uterus

\section{INTRODUCTION}

Most of the masses arising from the uterus during reproductive age group are benign myomas. But the dictum need not be true always. Here we are presenting a rare case of uterine metastasis from a rare malignancy in the abdominal cavity i.e. desmoplastic small round cell tumour.

\section{CASE REPORT}

A 22 year old primiparous lady, delivered 3 months ago by caesarean section for cephalopelvic disproportion and Fibroid complicating pregnancy, was referred to us with heavy bleeding per vaginum when she started her cycles postpartum. There was no primary postpartum haemorrhage and lochia was normal in quantity and duration. She gave a history of had been diagnosed to have small $2 \times 2 \mathrm{~cm}$ fibroid uterus 2 years ago while evaluating for infertility. Postpartum the size of the Uterus remained at 24 weeks size .She was referred to us for uterine artery embolisation. On examination, she was pale, per abdomen showed the uterus enlarged to 24 weeks size, On speculum cervical fibroid of size $6 \times 6 \mathrm{~cm}$ seen obliterating the posterior lip. Bleeding + per vaginal examination confirmed the findings. Haemogram revealed $\mathrm{Hb}$ of $6 \mathrm{gm} \%$. Ultrasound imaging showed $10 \times 12 \mathrm{~cm}$ posterior wall fibroid extending to the Cervix. After stabilizing with 2 pints of packed RBC transfusion, she was taken up for Uterine Artery Embolisation (UAE). Because of her age and postpartum status, UAE was chosen as the modality of treatment. She was symptom free for 2 months. Then on follow up after 2 months; clinically the fibroid size remained the same. MRI showed large heterogenous mass with calcification and haemorrhage in the posterior wall and a large fibroid in the posterior lip of cervix, cavity not delineated properly. An open myomectomy was planned. Intraoperatively fibroid was found to occupy the bulk of uterus with numerous seedling fibroids, encroaching the posterior wall (Figure 1). The appendix and large bowel was found to be adherent to the fibroid with numerous small masses, suspicious of wandering fibroids, along the common and internal iliac vessels. Hence it was converted to hysterectomy along with appendicectomy. Internal iliac ligation was done anticipating heavy bleeding. Histopathological examination revealed a poorly differentiated neoplasm. Tumour cells were positive for vimentin, desmin (paranuclear), focally for EMA, NSE; (Figure 2, 3) negative for CK, SMA, CD10, Myf 4, 
CD117, ER, PR. CD99, AFP-nonspecific staining. p53 $80-85 \%$ of cells show positivity. Ki 67 index-35-40\%. Findings were consistent with desmoplastic small round cell tumour. Cytogenetic study (t 11:22) was advised for confirmation. Postoperatively she was found to have two small masses in the left breast; trucut biopsy confirmed the metastasis from the same tumour. Medical Oncology opinion was sought. After discussing in the tumour board, chemotherapy was planned. Tumour markers were negative for BHCG, CEA. She had completed 6 cycles of chemotherapy with VAC-IE regimen. Follow up PET scan showed persisting disease in the iliac nodes and lung metastasis. Finally she succumbed to the disease after 18 months of diagnosis.

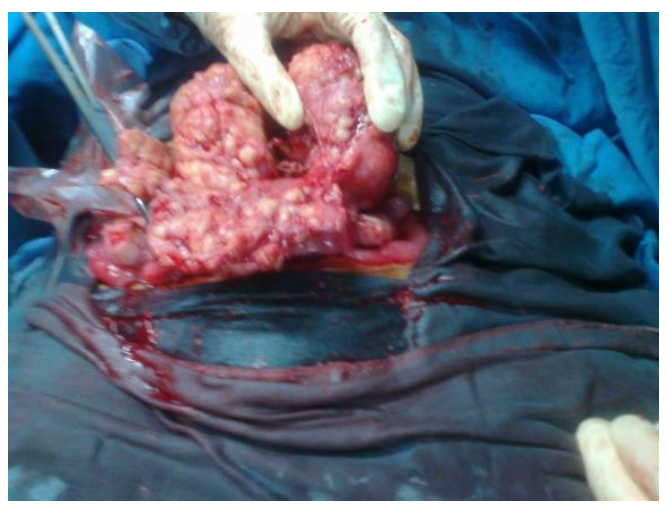

Figure 1: Peroperative picture of desmoplastic small round cell tumour.

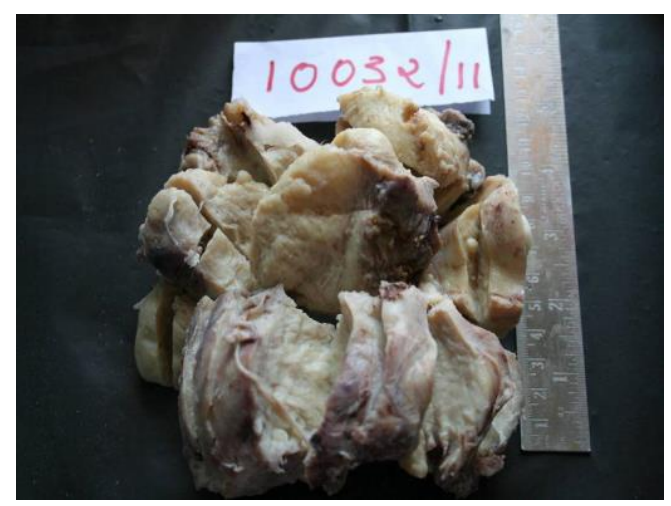

Figure 2: Cut section of the same mass.

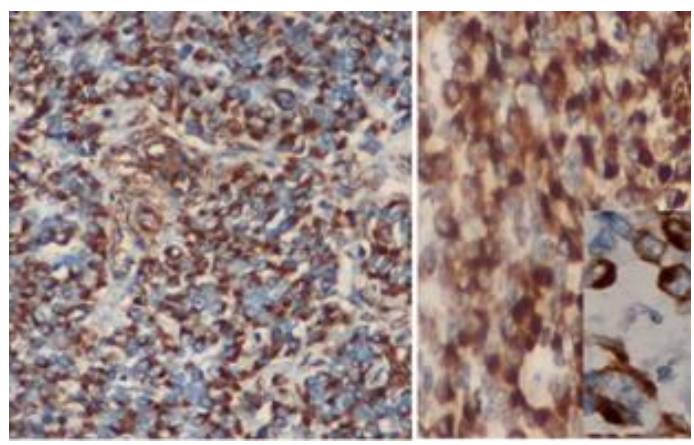

Figure 3: Immunohistochemical staining for vimentin and desmin.

\section{DISCUSSION}

Desmoplastic small round cell tumour is an aggressive neoplasm and is thought to originate from a mesothelial or submesothelial progenitor cell with the potential to undergo multilineage differentiation. Desmoplastic Small Round Cell Tumour (DSRCT) was first described in 1989 by Gerald and Rosai and Ordonez et al. It usually occurs in males during adolescence and early adulthood. Only a few hundred cases have been reported in worldwide literature. The male to female ratio is $4: 1{ }^{1}$

In most cases, DSRCT presents as an abdominal mass with peritoneal and omental implants. Other reported sites of disease include pleura, ethmoid sinuses, scalp, hand, posterior cranial fossa, pancreas, ovary, paratesticular and kidney. Visceral metastasis to uterus has not been described in the literature till now. Retrovesical and rectouterine peritoneum involvement has been described. Interestingly, DSRCT has been documented as an incidental finding during a cesarean section.

Associated symptoms may include crampy abdominal pain, weight loss and constipation. DSRCT is regional; the major bulk of these tumors is intraabdominal. More than $40 \%$ of patients have distant metastases at the time of diagnosis, mostly located in the liver, lungs, and lymph nodes.

These masses are frequently hypoechoic on ultrasound. CT scan is most often used for initial diagnosis that shows multiple peritoneal masses with regular contour situated within mesentery. The most common imaging finding of patients with intra-abdominal DSRCT was multiple peritoneal soft tissue masses, with a dominant mass in the retrovesical or rectouterine location in more than half of the cases. ${ }^{2}$ All tumors show inhomogeneous enhancement on CT. Punctate area of calcification can be seen. MRI findings include lesions with hyperintense T2 -weighted signal and an isointense signal on T1-weighted sequences. Haemorrhage and necrosis can be identified using varying signal intensities inside the mass. ${ }^{3}$ In patients with fibroid uterus who underwent uterine artery embolisastion, haemorrhagic necrosis can be found. But the loss of Junctional zone between endometrium and myometrium and heterogeneity point towards malignancy. The rarity of this tumour and the similarities it shares with the other small round cell tumours still make diagnosis challenging. However, distinctive cytological, histological, immunocytochemical and cytogenetic profiles have all been described for DSRCT. Histologic examination shows small cells that can be round, ovoid or spindled usually grouped in clumps, cords, nests or sheets. These cells contain hyperchromatic nuclei with condensed chromatin and eosinophilic cytoplasm. Extensive collagenous stroma is characteristic, and desmoplasia is a distinctive feature to this tumor. A polyphenotypic pattern of immunohistochemical markers has been described. DSRCT express antigens related to different cell lineages, 
including: epithelial-cytokeratin, epithelial membrane antigen, mesenchymal-desmin, vimentin. Neural-neuronspecific enolase, synaptophysin. ${ }^{4}$ The most specific diagnostic tool is the presence of the EWS-WT1 gene fusion that can be detected by RT-PCR and FISH. Treatment options include surgery, radiotherapy, chemotherapy with or without stem cell transplantation, and recently introduced molecularly targeted therapies. Unfortunately there is no standard therapeutic regimen described since no modality is clearly superior to any other. The 5 year survival rate is $15 \%$.

Funding: No funding sources

Conflict of interest: None declared

Ethical approval: Not required

\section{REFERENCES}

1. Stuart-Buttle CE, Smart CJ, Pritchard S, Martin D, Welch IM. Desmoplastic small round cell tumour: a review of literature and treatment options. Surg Oncol. 2008 Aug; 17(2):107-12.

2. Arora VC, Price AP, FlemingS, Sohn MJ, Magnan $\mathrm{H}$, La Quaglia MP, Abramson S. Characteristic imaging features of desmoplastic small round cell tumour. Peadiatr Radiol. 2013 Jan;43(1):93-102.

3. B. Kis, K. n O'Regan, A. Agoston, O. Javery, J. Jagannathan, N. H. Ramaiya. Imagimg of small round cell tumour in adults. Br J Radiol. 2012 Feb; 85(1010):187-92.

4. Lae ME, Roche PC, Jin L, Lloyd RV, Nascimento AG. Desmoplastic small round cell tumor: a clinicopathologic, immunohistochemical, and molecular study of 32 tumors. Am J Surg Pathol. $2002 \mathrm{Jul} ; 26(7): 823-35$.

DOI: $10.5455 / 2320-1770 . i j \operatorname{cog} 20140634$

Cite this article as: Kanthi JM, Sreedhar S, Nair

IR. Rare peritoneal tumour presenting as uterine fibroid. Int J Reprod Contracept Obstet Gynecol 2014;3:450-2. 